\title{
PORTAF - postoperative radiotherapy of non-small cell lung cancer: accelerated versus conventional fractionation - study protocol for a randomized controlled trial
}

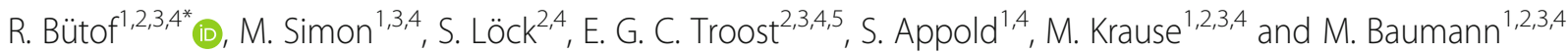

\begin{abstract}
Background: In early-stage non-small cell lung cancer (NSCLC) without affected lymph nodes detected at staging, surgical resection is still the mainstay of treatment. However, in patients with metastatic mediastinal lymph nodes (pN2) or non-radically resected primary tumors (R1/R2), postoperative radiotherapy (possibly combined with chemotherapy) is indicated. So far, investigations about time factors affecting postoperative radiotherapy have only examined the waiting time defined as interval between surgery and start of radiotherapy, but not the overall treatment time (OTT) itself. Conversely, results from trials on primary radio(chemo)therapy in NSCLC show that longer OTT correlates with significantly worse local tumor control and overall survival rates. This time factor of primary radio(chemo)therapy is thought to mainly be based on repopulation of surviving tumor cells between irradiation fractions. It remains to be elucidated if such an effect also occurs when patients with NSCLC are treated with postoperative radiotherapy after surgery (and chemotherapy). Our own retrospective data suggest an advantage of shorter OTT also for postoperative radiotherapy in this patient group.

Methods/design: This is a multicenter, prospective randomized trial investigating whether an accelerated course of postoperative radiotherapy with photons or protons (7 fractions per week, 2 Gy fractions) improves locoregional tumor control in NSCLC patients in comparison to conventional fractionation (5 fractions per week, 2 Gy fractions). Target volumes and total radiation doses will be stratified in both treatment arms based on individual risk factors.

Discussion: For the primary endpoint of the study we postulate an increase in local tumor control from 70\% to 85\% after 36 months. Secondary endpoints are overall survival of patients; local recurrence-free and distant metastases-free survival after 36 months; acute and late toxicity and quality of life for both treatment methods.
\end{abstract}

Trial registration: ClinicalTrials.gov, NCT02189967. Registered on 22 May 2014.

Keywords: Postoperative radiotherapy, Non-small-cell lung cancer (NSCLC), Fractionation, Acceleration, Randomized clinical trial, Phase II trial, Overall treatment time, Time factor

\footnotetext{
*Correspondence: rebecca.buetof@uniklinikum-dresden.de

'Department of Radiotherapy and Radiation Oncology, Faculty of Medicine and University Hospital Carl Gustav Carus, Technische Universität Dresden, Dresden, Germany

${ }^{2}$ OncoRay - National Center for Radiation Research in Oncology, Faculty of Medicine and University Hospital Carl Gustav Carus, Technische Universität Dresden, Helmholtz-Zentrum Dresden - Rossendorf, Germany

Full list of author information is available at the end of the article
} 


\section{Background}

Non-small cell lung cancer (NSCLC) is one of the world's most common malignant diseases [1,2]. Despite the establishment of new systemic treatment options and improvement of treatment techniques, the prognosis of the patients is still poor with an average overall survival between $5.5 \%$ and $15.7 \%$ after 5 years $[1,2]$.

In early-stage NSCLC surgical resection is still the mainstay of treatment [2]. The role of postoperative radiotherapy (PORT) has been investigated in a number of studies and the results were summarized in two metaanalyses $[3,4]$. These studies are extremely heterogeneous in terms of radiation dose, fractionation schedules and the quality of the irradiation technique. Because of this heterogeneity, clinical interpretation remains controversial. It appears clear that patients after complete surgical resection (R0) and with limited lymph node involvement ( $\mathrm{N} 0$ or $\mathrm{N} 1$ ) do not benefit from postoperative radiation [2-4]. In contrast, there is evidence for improved local tumor control and possibly improved overall survival of patients with regionally advanced tumors (pN2) after PORT [3, 5-8]. Therefore, PORT is offered by many centers in this situation.

Based on results of randomized clinical trials and their meta-analyses $[9,10]$, the S3 guideline released by the German Cancer Society and the German Respiratory Society recommends the application of postoperative adjuvant chemotherapy for patients with stage III NSCLC [2], which increases survival rates after 5 years by approximately $4 \%$ [9]. The guideline also recommends consideration of PORT, which should start about 4 weeks after the end of chemotherapy [2]. The application after the end of chemotherapy leads to a delayed start of radiotherapy and a prolonged total treatment time as compared to earlier treatment concepts without chemotherapy. From a radiobiological point of view, this could lead to proliferation of tumor cells (repopulation) before the start of radiotherapy and thus to reduced locoregional control rates, at least in non- and low responders to chemotherapy. However, because of a stimulated accelerated repopulation of surviving tumor cells during chemotherapy, a reduced efficiency of PORT could arise for chemotherapy responders, too [11]. Thus far, investigations about the time factor of PORT of NSCLC patients have only examined waiting periods between surgery and the start of radiotherapy outside of sequential chemo-radiotherapy concepts. These retrospective studies have never proved a survival benefit for patients with shorter waiting periods between surgery and the start of radiotherapy $[12,13]$.

Randomized trials and their meta-analysis show that a longer overall treatment time (OTT) of primary radiotherapy correlates with significantly worse survival rates and local tumor control rates [14-18]. For PORT, a retrospective analysis of our own patient data indicates that such a time factor may also play an important role for NSCLC patients in the adjuvant situation [13]. Moreover, an explorative analysis based on stratification criteria of patients with a primary radiotherapy treatment within the CHARTWEL study shows that patients who received chemotherapy before radiotherapy demonstrated a significantly improved local tumor control with an HR of 0.5 after accelerated radiotherapy [16]. It remains to be elucidated if such an effect also occurs when patients are treated with PORT after chemotherapy. The aim of the present study is to investigate whether there is an improvement of local tumor control when using accelerated radiotherapy in the postoperative situation in NSCLC compared to conventional fractionation. Secondary endpoints include the evaluation of quality of life, overall survival, occurrence of distant metastases and acute and late radiation-induced side effects.

\section{Methods/design}

This is a multicenter, prospective randomized phase II trial investigating whether accelerated postoperative radiotherapy [7 fractions per week, 2 Gray (Gy) fractions] may improve locoregional tumor control in nonsmall cell lung cancer (NSCLC) in comparison to conventional fractionation (5 fractions per week, 2 Gy fractions). Target volumes and total radiation doses will be stratified in both treatment arms based on individual risk factors (details see below in the Radiotherapy section). In both treatment arms irradiation may be delivered with photons or protons.

\section{Recruitment, randomization, and workflow}

Patients will be informed about the study by the treating radiation oncologist. All patients with signed informed consent are included in the study. The following clinical examinations have to be performed before randomization: complete staging including fluorodeoxyglucose positron emission tomography/computed tomography (FDG-PET/CT) or alternatively contrastenhanced CT chest/abdomen and bone scintigraphy; magnetic resonance imaging (MRI) of the skull only in cases of suspected brain metastases; blood analyses and postoperative pulmonary function test. For patients who received adjuvant chemotherapy, the entire staging should be repeated prior to start of radiotherapy.

After finishing all obligatory pre-treatment assessments, randomization will be done electronically via RadPlanBio [19]. Patients will be stratified by the following criteria: staging with or without PET-CT; resection status R1 or R0; and the respective study center. The result of randomization will be available immediately after the patient has been registered in RadPlanBio. Patients with macroscopic tumor can be treated in stratum " $R$ " 
and will receive conventionally fractionated radiation therapy to a total dose of 66 Gy according to the current clinical guideline. Figure 1 shows a flowchart of the study.

Inclusion criteria:

- histologically confirmed non-small cell lung cancer

- surgery performed with curative intention

- postoperative indication for radiotherapy (> pN1 and/or R1)

- R2 resection or recurrence postoperatively/after adjuvant chemotherapy diagnosed by the restaging imaging

- no distant metastases (M0)

- patient $\geq 18$ years old

- good general health condition [Eastern Cooperative Oncology Group (ECOG) score 0 or 1]

- signed informed consent

- appropriate compliance to ensure follow-up visits

- women of childbearing age: adequate contraception

Exclusion criteria:

- histologically confirmed small cell lung cancer

- distant metastases (M1)

- patient is unable to understand reason, purpose, and side effects of this study

- previous ( $<5$ years) or synchronous malignancy (except in situ carcinomas, basal cell carcinoma, early-stage skin cancer)

- for proton therapy: cardiac pacemaker

- previous radiotherapy in the thoracic or lower head and neck region

- pregnancy or lactation

- participation in another clinical intervention trial or not completed follow-up of a clinical intervention study (except psychological studies, supportive, or observational studies)

\section{Primary and secondary endpoints}

The primary endpoint of this study is locoregional tumor control 36 months after start of radiotherapy comparing an accelerated irradiation schedule (7 fractions per week, 2 Gy single dose) with the standard conventional fractionation schedule (5 fractions per week, 2 Gy single dose) for POST in patients with NSCLC.

Secondary endpoints contain overall survival of patients; local recurrence-free and distant metastases-free survival 36 months after start of radiotherapy; acute and late toxicity and quality of life for both treatment methods. All primary and secondary endpoints will be compared for the photon and proton-treated patients.

Clinical follow-up examinations with additional imaging scans (FDG-PET/CT or CT thorax/abdomen) are scheduled every 3 months within the first 3 years and every 6 months within the following 2 years. During therapy and at the follow-up visits, scoring of side effects will be performed according to Common Terminology Criteria for Adverse Events (CTC-AE) 4.0. Quality of life will be assessed additionally by the European Organisation for the Research and Treatment of Cancer (EORTC) questionnaires QLQ-C30 and QLQ-LC13 before and at the end of irradiation and at each follow-up visit.

The trial design and protocol adhere to Standard Protocol items: Recommendations for Interventional Trials (SPIRIT) criteria (www.spirit-statement.org); the SPIRIT checklist and figure can be found as Additional file 1: Table S1 and Additional file 2: Figure S1.

\section{Biometrical design}

The primary endpoint, locoregional tumor control after 36 months, will be investigated in 1:1 randomized patient cohorts treated with accelerated or conventional fractionated irradiation. Our hypothesis is an improvement of $15 \%$ after 3 years in the accelerated treatment arm (7 fractions per week) in the group of R0- or R1-



Fig. 1 Flowchart 
resected patients; i.e., an increase of the local tumor control rate from $70 \%$ to $85 \%$.

With accelerated irradiation, treatment time will be shortened from 33 days to 24 days if a total dose of $50 \mathrm{~Gy}$ is applied. If a total dose of 60 Gy is applied, treatment time will be reduced from 40 days to 29 days. On average, this means a reduction of 10 treatment days and with the assumption of gaining $0.6 \mathrm{~Gy}-0.8 \mathrm{~Gy}$ of dose per day (by preventing repopulation), this corresponds to an increase of the biological dose of $10-16 \%$ in the accelerated arm, depending on the prescribed total dose. Conservatively assuming a $y$-factor $=1$ (steepness of the dose-response curve), the locoregional tumor control rate would increase by $10-16 \%$ from $70 \%$ to $80-86 \%$.

Assuming $\alpha$ is 0.05 , a rise of $15 \%$ can be detected with the power of $80 \%$ if 154 patients are treated in every arm. For this calculation a Cox proportional hazard model was assumed with hazard ratio 0.475 , overall probability of event $22.5 \%$ and $1: 1$ randomization [20]. Furthermore, a drop-out rate of $10 \%$ of the participants has been used.

The " $R$ " stratum, which contains patients with macroscopic remaining tumor or recurrences detected in preradiotherapeutic staging, was not included in the statistical sample size calculation.

\section{Radiotherapy}

For radiotherapy planning purposes, all patients should undergo an FDG-PET/CT in treatment position. Alternatively, radiotherapy planning can be performed solely based on $\mathrm{CT}$ in treatment position. The acquisition of a four-dimensional computed tomography (4D-CT) for radiotherapy planning purposes is favored, but not mandatory for the treatment with photons. For proton therapy, the implementation of a $4 \mathrm{D}-\mathrm{CT}$ is indispensable to calculate the mobility of the target volume and to consider it in the radiation treatment planning. On the basis of the planning $\mathrm{CT}$, the clinical target volume (CTV) and the organs at risk are contoured. In tumors of the right lung, the CTV includes the mediastinal lymph node stations $2 \mathrm{R}, 4 \mathrm{R}$, and 7 as well as the right hilum. In tumors of the left lung, the CTV includes the lymph node stations $2 \mathrm{~L}, 4 \mathrm{~L}, 5,6$, and 7 as well as the left hilum. Additionally, in tumors of the left inferior lobe levels $4 R / 2 R$ are to be included. Moreover, the surgical report and surgical clips are to be considered. The CTV will subsequently be expanded to the planning target volume for photon therapy taking into account the institution's guidelines. For proton therapy individual range uncertainties will be considered. The contouring has been standardized with a mandatory dummy run for all participating centers.
Irradiation planning will be done three dimensionally with the help of a clinically licensed treatment planning system. Every patient undergoing photon therapy will be treated with an image-guided conformal radiation technique [three-dimensional conformal radiation therapy (3D-CRT), intensity-modulated radiation therapy (IMRT)]. For proton radiotherapy, patient positioning will be performed based on orthogonal X-ray and correction for the bony anatomy. Target coverage and dose to organs at risk will be assessed by at least one physician and one physicist prior to the start of treatment. Constraints will be according to current clinical guidelines based on QUANTEC. All patients who are treated with protons receive therapy with passively scattered or actively scanned protons.

Patients will be treated either with an accelerated irradiation schedule or with conventional fractionation (7 or 5 fractions per week, 2 Gy fractions, respectively). The mediastinal target volume will be defined according to clinical guidelines and irradiated up to a total dose of 50 Gy. In addition, boost irradiation of 10 Gy (2 Gy single dose) will be applied in cases of a partial resection (R1 situation) or extracapsular tumor spread (ECE) (total dose 60 Gy). For patients with an R2 situation or a detected recurrence postoperatively/after adjuvant chemotherapy (" $R$ " stratum), the boost dose is escalated to $16 \mathrm{~Gy}$. In this group, the total dose of thus $66 \mathrm{~Gy}$ is administered with standard fractionation (5 fractions/week).

Accelerated radiotherapy is performed by application of a second fraction on 2 (not consecutive) days every week and/or an additional irradiation at weekends. It is mandatory to have an interfraction interval of at least 6 , preferably even 8 hours.

\section{Discussion}

The presented phase II randomized trial evaluates for the first time if an accelerated postoperative irradiation schedule in NSCLC patients results in higher local tumor control rates compared to conventional fractionation. Several arguments support our hypothesis: a time factor, i.e., an impaired local tumor control after longer OTT of radiotherapy has been shown for primary radiotherapy schedules in NSCLC [14-18]; repopulation as the main reason for this time factor has been shown to be potentially induced by chemotherapy and this in turn is applied upfront to radiotherapy in the adjuvant situation [11]; our own retrospective data show impaired local tumor control rates after longer versus shorter overall treatment times of adjuvant radiotherapy [13]. In detail, a significant correlation between overall radiation treatment time and overall survival $(61 \%$ vs. $36 \%$ at 2 years, $p=0.001)$, relapse-free ( $90 \%$ vs. $51 \%$ at 2 years, $p=0.002)$ and metastases-free survival $(54 \%$ vs. $43 \%$ at 2 years, $p=0.015$ ) was found in this study. 
For acceleration of radiotherapy, the present study uses standard doses of 2 Gy per fraction and increases the radiation dose per week to $14 \mathrm{~Gy}$. This is in contrast to previous acceleration trials on primary radiotherapy, where accelerated hyperfractionated schedules have been used with up to 3 irradiation fractions of 1.5 Gy per day on 5-7 days per week $[16,17]$. The reason for the additional use of hyperfractionation was that extremely short treatment schedules have been used with a high dose-intensity, i.e., high dose per day or per week. Hyperfractionation instead of the application of the whole daily dose in one fraction leads here to better sparing of normal tissues due to repair and recovery between the fractions. Based on our own retrospective data the present trial uses a more moderate acceleration schedule. The application of 2 Gy per fraction is not only easier to apply in daily workflows of radiotherapy departments, it is also coming closer to recent radiotherapy schedules applied in dose escalation trials that are using doses of $>2$ Gy per fraction [21].

In addition, further optimization of postoperative irradiation in NSCLC patients may include the use of proton or heavier ion radiotherapy, which may enable better sparing of normal tissues but would hardly be applicable in hyperfractionated schedules. Therefore, the influence of proton therapy on early and late toxicity in the adjuvant situation in NSCLC patients will be investigated in this trial.

\section{Trial status}

Patient accrual started in November 2014 and is currently ongoing. At present, 14 centers participate in this study (Germany and Poland).

\section{Additional files}

Additional file 1: SPIRIT 2013 Checklist: Recommended items to address in a clinical trial protocol and related documents*. (DOC $122 \mathrm{~kb}$ )

Additional file 2: SPIRIT figure. (DOC $49 \mathrm{~kb}$ )

\begin{abstract}
Abbreviations
3D-CRT: Three-dimensional conformal radiation therapy; 4D-CT: Fourdimensional computed tomography; CT: Computed tomography; CTCAE: Common Terminology Criteria for Adverse Events; CTV: Clinical target volume; FDG: Fluorodeoxyglucose; Gy: Gray; IMRT: Intensity-modulated radiation therapy; MRI: Magnetic resonance imaging; NSCL: Non-small-cell lung carcinoma; OTT: overall treatment time; PET: Positron emission tomography; PORT: Postoperative radiotherapy
\end{abstract}

\section{Acknowledgements}

Julia Thiele, Dr. Annika Jakobi and Dr. Cordelia Hoinkis are acknowledged for their efforts in quality assurance and homogenization of the irradiation technique.

\section{Funding}

The study will be funded by institutional funding within the German Cancer Consortium (DKTK), partner site Dresden.
Availability of data and materials

Not applicable.

\section{Data monitoring committee}

The Data Monitoring Committee (DMC) consists of four independent members: three clinicians with experience in the study-specific indication area of radiotherapy, and one biometrician. The members of the DMC are independent from the sponsor and have declared that there exists no scientific or financial conflict of interest.

The DMC consults and supports the principal investigator and the protocol committee in regards of patient safety in the context of the clinical trial on hand. To this end, the DMC regularly reviews the results of safety analysis, quality controls, evaluation of toxicity and their impact on the benefit-risk assessment for the patient and the acceptability of the continuation of the study therapy. In addition, the DMC might review the validity and integrity of the data and evaluate the conduct of the study on this basis. To ensure data quality and consistency, internal quality control measures will be implemented by the senior clinical trial center. All reports will be provided to the DMC. The DMC meets during the conduct of the trial at least once a year and additionally if required.

In the working procedures guideline for the DMC consistence, responsibilities and details of the applied working procedures including documentation and reporting are defined.

\section{Authors' contributions}

$\mathrm{RB}$ and $\mathrm{MB}$ drafted the trial protocol and the manuscript. SL created the statistics. MS helped to draft the trial protocol, MK and ET revised the trial protocol and both authors improved the manuscript. MK, MB, SL, ET, MS, and SA provided trial ideas, and reviewed the trial protocol and manuscript. All authors are members of the protocol committee and read and approved the trial protocol and the final manuscript.

\section{Ethics approval and consent to participate}

The study (version 2.0 from 18 January 2017) was approved by the Ethics Committee of the Technische Universität Dresden, EK149042014. Every patient must give his/her written consent to participate in the study.

Consent for publication

Not applicable.

\section{Competing interests}

The authors declare that they have no competing interests.

\section{Publisher's Note}

Springer Nature remains neutral with regard to jurisdictional claims in published maps and institutional affiliations.

\section{Author details}

'Department of Radiotherapy and Radiation Oncology, Faculty of Medicine and University Hospital Carl Gustav Carus, Technische Universität Dresden, Dresden, Germany. ${ }^{2}$ OncoRay - National Center for Radiation Research in Oncology, Faculty of Medicine and University Hospital Carl Gustav Carus, Technische Universität Dresden, Helmholtz-Zentrum Dresden - Rossendorf, Germany. ${ }^{3}$ German Cancer Consortium (DKTK), partner site Dresden and German Cancer Research Center (DKFZ), Heidelberg, Germany. ${ }^{4}$ National Center for Tumor Diseases (NCT), Partner Site Dresden, Dresden, Germany. ${ }^{5}$ Helmholtz-Zentrum Dresden - Rossendorf, Institute of Radiooncology OncoRay, Dresden, Germany.

Received: 20 April 2017 Accepted: 24 November 2017 Published online: 20 December 2017

\section{References}

1. Alberg AJ, Ford JG, Samet JM. Epidemiology of lung cancer: ACCP evidencebased clinical practice guidelines (2nd edition). Chest. 2007;132:29S-55.

2. Goeckenjan G, Sitter H, Thomas M, et al. Prevention, diagnosis, therapy, and follow-up of lung cancer: interdisciplinary guideline of the German Respiratory Society and the German Cancer Society. Pneumologie. 2011;65:39-59.

3. Group PM-aT. Postoperative radiotherapy in non-small-cell lung cancer: systematic review and meta-analysis of individual patient data from nine randomised controlled trials. Lancet. 1998;352:257-63. 
4. Le Pechoux C. Role of postoperative radiotherapy in resected non-small cell lung cancer: a reassessment based on new data. Oncologist. 2011;16:672-81.

5. Dai H, Hui Z, Ji W, et al. Postoperative radiotherapy for resected pathological stage IIIA-N2 non-small cell lung cancer: a retrospective study of 221 cases from a single institution. Oncologist. 2011;16:641-50.

6. Du F, Yuan Z, Wang J, et al. The role of postoperative radiotherapy on stage N2 non-small cell lung cancer. Zhongguo Fei Ai Za Zhi. 2009;12:1164-8.

7. Lally BE, Zelterman D, Colasanto JM, Haffty BG, Detterbeck FC, Wilson LD. Postoperative radiotherapy for stage II or III non-small-cell lung cancer using the surveillance, epidemiology, and end results database. J Clin Oncol. 2006; 24:2998-3006.

8. Mantovani C, Giaj Levra N, Filippi AR, et al. Postoperative radiotherapy for patients with completely resected pathologic N2 non-small-cell lung cancer: a retrospective analysis. Clin Lung Cancer. 2013;14:194-9.

9. Arriagada R, Bergman B, Dunant A, Le Chevalier T, Pignon JP, Vansteenkiste J. Cisplatin-based adjuvant chemotherapy in patients with completely resected non-small-cell lung cancer. N Engl J Med. 2004;350:351-60.

10. Arriagada R, Auperin A, Burdett $\mathrm{S}$, et al. Adjuvant chemotherapy, with or without postoperative radiotherapy, in operable non-small-cell lung cancer: two meta-analyses of individual patient data. Lancet. 2010;375:1267-77.

11. Bütof R, Baumann M. Time in radiation oncology - keep it short! Radiother Oncol. 2013;106(3):271-5.

12. Wurschmidt $F$, Bunemann $H$, Ehnert $M$, Heilmann HP. Is the time interval between surgery and radiotherapy important in operable nonsmall cell lung cancer? A retrospective analysis of 340 cases. Int J Radiat Oncol Biol Phys. 1997:39:553-9.

13. Bütof R, Kirchner K, Appold S, et al. Potential clinical predictors of outcome after postoperative radiotherapy of non-small cell lung cancer. Strahlenther Onkol. 2014;190(3):263-9.

14. Mauguen A, Le Pechoux C, Saunders MI, et al. Hyperfractionated or accelerated radiotherapy in lung cancer: an individual patient data metaanalysis. J Clin Oncol. 2012;30:2788-97.

15. Ball D, Bishop J, Smith J, et al. A randomised phase III study of accelerated or standard fraction radiotherapy with or without concurrent carboplatin in inoperable non-small cell lung cancer: final report of an Australian multicentre trial. Radiother Oncol. 1999;52:129-36.

16. Baumann $M$, Herrmann $T$, Koch $R$, et al. Final results of the randomized phase III CHARTWEL-trial (ARO 97-1) comparing hyperfractionatedaccelerated versus conventionally fractionated radiotherapy in non-small cell lung cancer (NSCLC). Radiother Oncol. 2011;100:76-85.

17. Saunders M, Dische S, Barrett A, Harvey A, Gibson D, Parmar M. Continuous hyperfractionated accelerated radiotherapy (CHART) versus conventional radiotherapy in non-small-cell lung cancer: a randomised multicentre trial. CHART Steering Committee. Lancet. 1997;350:161-5.

18. Sause WT, Scott C, Taylor S, et al. Radiation Therapy Oncology Group (RTOG) 88-08 and Eastern Cooperative Oncology Group (ECOG) 4588: preliminary results of a phase III trial in regionally advanced, unresectable non-small-cell lung cancer. J Natl Cancer Inst. 1995;87:198-205.

19. Skripcak T, Just U, Simon M, et al. Towards distributed conduction of large scale studies in radiation therapy and oncology: open source system integration approach. IEEE J Biomed Health Inform 2016; epub. doi:10.1109/ JBHI.2015.2450833.

20. Chow S, Shao J, Wang H. Sample size calculations in clinical research. 2nd ed. Boca Raton: Chapman \& Hall/CRC Biostatistics Series; 2008. p. 177

21. Walraven I, van den Heuvel M, van Diessen J, et al. Long-term follow-up of patients with locally advanced non-small cell lung cancer receiving concurrent hypofractionated chemoradiotherapy with or without cetuximab. Radiother Oncol. 2016;118(3):442-6.

\section{Submit your next manuscript to BioMed Central and we will help you at every step:}

- We accept pre-submission inquiries

- Our selector tool helps you to find the most relevant journal

- We provide round the clock customer support

- Convenient online submission

- Thorough peer review

- Inclusion in PubMed and all major indexing services

- Maximum visibility for your research

Submit your manuscript at www.biomedcentral.com/submit

) Biomed Central 